\section{A Compact Dual-polarized 4-port Eleven Feed with High Sensitivity for Reflectors over 0.35-1.05 GHz}

Jian Yang, Miroslav Pantaleev, Bhushan Billade, Marianna Ivashina, Tobia Carozzi, Leif Helldner, Magnus Dahlgren

\begin{abstract}
We present significant improvements to the circular Eleven feed technology for a dual reflector system operating over 0.35-1.05 GHz as a backup for the Square-KilometerArray (SKA) project Band 1. In this work, the number of the feed ports is reduced to 4 from the previous 8 for dual polarization by using a novel geometry at the center. The design is carried out by optimizing with a social civilization algorithm. The resulting improvements include a reflection coefficient below $-12 \mathrm{~dB}$, an aperture efficiency above $70 \%$ at the upper end of the band, a maximum cross-polar level under $-15 \mathrm{~dB}$, and an ohmic loss about $0.05 \mathrm{~dB}$. A prototype based on this design has been manufactured and the design simulations have been verified against measurements. A simulated sensitivity of the dual-reflector receiver system for the SKA project based on the measured data is also presented in the paper.
\end{abstract}

Index Terms-Eleven feed technology, high aperture efficiency, high sensitivity.

\section{INTRODUCTION}

Eleven feed technology has received significant attention recently in ultra-wideband (UWB) radio telescopes [1]-[3], such as the SKA (square kilometer array) and VGOS (VLBI2010 Global Observing System) projects. In this paper, we present new improvements to this technology.

Next generation radio telescopes can greatly benefit from a UWB system with a high antenna efficiency and low receiving noise level. Several UWB feed technologies for reflector antennas have been developed with great advantages over conventional feed technologies. The quadruple-ridge flared horns (QRFH) [4] offers beamwidth flexibility and excellent impedance match to standard 50-Ohm single-ended low-noise amplifiers (sLNAs). Some drawbacks of this technology, such as a strong variation of H-plane beamwidth with freuency and relatively high cross-polarization level, have been remedied to some extent by the quadraxial feeding in combination with differential low-noise amplifiers (dLANs) [5]. Other novel UWB feed technologies include the sinuous antenna on an inverted cone [6], the quasi-self-complementary feed [7], and the Dyson conical quad-spiral feed [8].

The noise performance of a radio telescope strongly depends upon the selected antenna feeding concept, the sLNA vs. dLNAs choice and cooling implementation. As has been studied in [9], for frequencies above $2 \mathrm{GHz}$, a fully integrated cryogenic feed-LNA solution is feasible and would obviously provide the best sensitivity. At lower frequencies, a reduced complexity of the feeding implementation and hence improved

J. Yang and M. Ivashina are with the Dept. of Signals and Systems, M. Pantaleev, B. Billade, T. Carozzi, L. Helldner and M. Dahlgren are with the Dept. of Earth and Space Sciences (Onsala Space Observatory), all at Chalmers University of Technology, S-41296 Gothenburg, Sweden. e-mail: jian.yang@chalmers.se.

Manuscript received 2015.

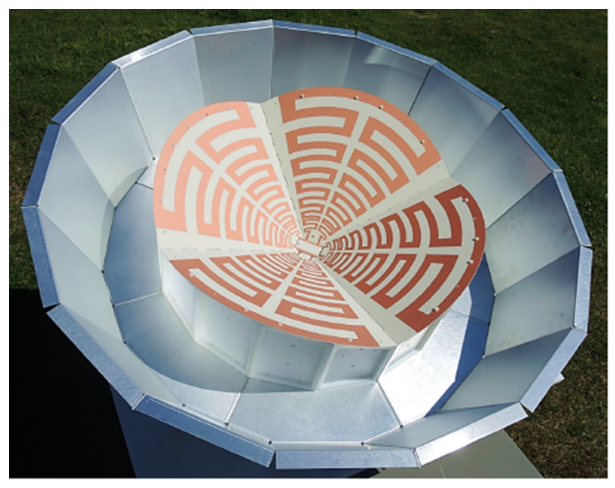

Fig. 1. Prototype of the Eleven feed over $0.35-1.05 \mathrm{GHz}$ with the size of $745 \mathrm{~mm}$ in diameter and $241 \mathrm{~mm}$ in height, with a metal cone which has a size of $1200 \mathrm{~mm}$ in diameter and $250 \mathrm{~mm}$ in height.

antenna impedance match would minimize the noise level. This low-frequency system integration option represents the motivation for the current work.

The Eleven feed technology has some unique characteristics: nearly constant beam width, fixed phase center location, low cross-polar level, low ohmic loss and a compact size [10], [11]. However, the previously developed Eleven feeds had 8 ports for dual polarization, which required either 8 sLNAs or 4 dLNAs for a dual polarized receiver system. All LNAs are required to have an identical performance over operating time in order to have a stable performance, which may cause a high cost.

A new geometrical structure, the center-bridge connection, is applied in this work for reducing the port number by a factor of 2 and increasing the isolation between two polarizations. A brief report on this solution with simulation results has been outlined in [12]. Here, the detailed design method and analysis with measurement data of a manufactured prototype shown in Fig. 1 are described. This solution, together with the use of a Social Civilization Algorithm (SCA) optimization scheme, improves the feed performance considerably.

\section{New Geometry of the Center Connection}

A direct center connection between two petals of dipole arrays was used in [10] for linearly polarized Eleven feed. Then, the same simple connections orthogonally with different heights above the ground plane for dual polarization were tested in [13]. However, the cross polar level in radiation was found to increase due to the capacitive coupling over the overlap area of the transmission twin-lines of the two polarizations. This reduced the polarization purity of signals at the receiver ends.

In order to solve this problem, a center-bridge geometry, inspired by highway overpass, is introduced in this work, see Fig. 2. The main idea is to isolate the two polarization feedings (removing the mutual coupling) by adding a metal bridge as an extra ground plane: Pol A (the polarization at the lower layer) is fed by two coax-cables with their inner conductors connected to twin-line A and outer conductors connected to the main ground plane of the feed; while Pol B (the polarization at the upper layer) is fed by other two coax-cables with their 


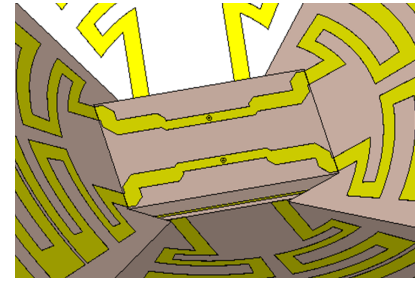

(a)

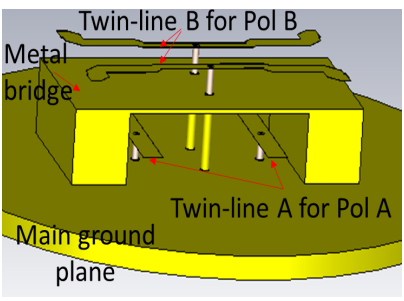

(b)
Fig. 2. New center-bridge geometry: (a) overview; (b) a clarified show of the structure by hiding the substrate and dipole petals.

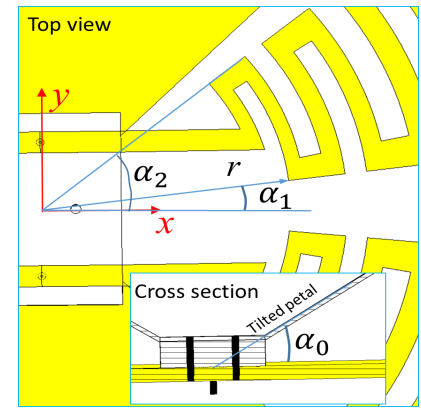

(a)

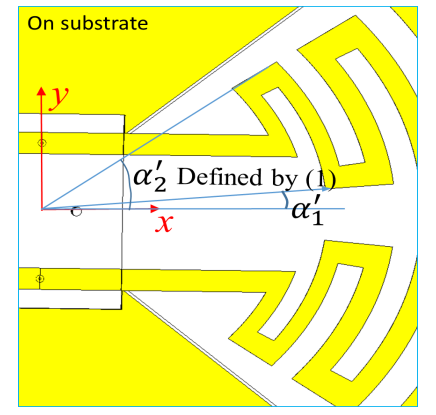

(b)
Fig. 3. New circular dipole modeling: (a) top view when petals are tilted, and (b) dipoles on substrate by (1).

inner conductors connected to twin-line B above the metal bridge and outer conductors connected to the bridge which is also connected to the main ground plane. By this arrangement, the feedings of the two different polarizations are isolated by the outer conductors of Pol B and the metal bridge.

Since the center geometries are different for Pol A and Pol $B$, the twin-lines at the center are different: straight lines for Pol A and step-transformer-type lines for Pol B, as shown in Fig. 2, and their dimensions are included in the optimization.

\section{Modeling AND Optimization}

The circular Eleven feed is used in this design due to its high $\mathrm{BOR}_{1}$ efficiency [3], [14]. A more accurate modeling method than that used in [3] is introduced in this work for CST MS [15] modeling, which is summarized in Fig. 3. The circular arms of folded dipoles are defined in the $x-y$ plane in such a way that their projections are truly circular when the petal of a folded dipole array on a substrate board is tilted at an angle $\alpha_{0}$ over $x$ - $y$ plane. The coordinates $\left(x_{\mathrm{arm}}, y_{\mathrm{arm}}\right)$ of curved arms are defined by

$$
\begin{aligned}
& x_{\text {arm }}=\frac{r \cdot \sin \varphi}{\cos \alpha_{0}} \\
& y_{\text {arm }}=r \cdot \cos \varphi
\end{aligned}
$$

in between $\alpha_{1}^{\prime}$ and $\alpha_{2}^{\prime}$, where

$$
\begin{aligned}
& \alpha_{1}^{\prime}=\operatorname{atan}\left(\cos \alpha_{0} \cdot \tan \alpha_{1}\right) \\
& \alpha_{2}^{\prime}=\operatorname{atan}\left(\cos \alpha_{0} \cdot \tan \alpha_{2}\right),
\end{aligned}
$$

with $r$ the radius of a circle, $\alpha_{0}$ tilted angle of the dipole petal, and angles $\alpha_{1}, \alpha_{2}, \alpha_{1}^{\prime}$ and $\alpha_{2}^{\prime}$ as shown in Fig. 3. By using this geometric description, the cut edges of the petals in

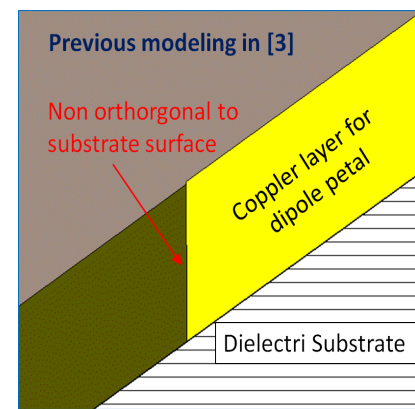

(a)

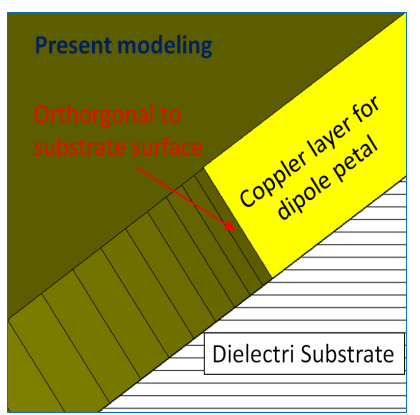

(b)
Fig. 4. Cross section of dipole petals and substrate: (a) previous modeling in [3] where the edges of dipole petals are not orthogonal to the surface of the tilted substrate, and (b) present modeling by (1).

CST model are orthogonal to the surface of the substrate as shown in Fig. 4, which represents the case when the petals are fabricated by PCB (printed circuit board) etching technique.

A social civilization algorithm (SCA) [16] was used to the design in this work with our own Matlab script. Compared to the Genetic Algorithm used in [17], SCA is usually faster to achieve convergency, more likely to find global optimum in the case of having multiple objectives as goal function, and simpler to implement in coding.

The primary design goal for the SKA is to maximize the receiving sensitivity, i.e. the ratio of antenna effective area to system noise temperature $\left(A_{e} / T_{\text {sys }}\right)$. The SKA baseline design represents an off-set Gregorian reflector system [18] equipped with several feeds operating at 5 bands from 0.35 to $13.8 \mathrm{GHz}$. The feed in this work is designed for the low frequency band (0.35-1.05 GHz).

In order to reduce the computation time for a feasible optimization, the feed was optimized for the case of an axisymmetric single-reflector system. The optimization objective is to realize an optimal illumination of the reflector with a given subtended angle (leading to high aperture efficiency and low spillover loss) and minimum reflector coefficient (reducing the system noise temperature contribution due to the impedance mismatch with the LNAs), while maintaining compact and low-profile dimensions to avoid the unwanted interaction effects with the reflector antenna structure. We define a figure of merit $S_{\text {app }}$ for the optimization as

$$
S_{\mathrm{app}}=\frac{e_{\mathrm{ap}}}{1-e_{\mathrm{sp}}}
$$

where $e_{\mathrm{ap}}$ is the aperture efficiency and $e_{\mathrm{sp}}$ the spillover efficiency of the feed when it feeds a primary reflector, because it needs to simulate only the feed alone without including the reflector system to get $S_{\text {app }}$.

The social behavior genes of an individual are defined by its geometrical parameters of the feed, similar to those in [3]. The fitness value $G_{\text {fitness }}$ of each individual is evaluated by a similar method to that used in [19], which can be expressed by

$$
G_{\text {fitness }}=1-\sqrt{\beta_{1}\left|S_{11}\right|^{2}+\beta_{2}\left(\frac{S_{\mathrm{app}}-S_{\mathrm{appmax}}}{S_{\mathrm{appmax}}}\right)^{2}},
$$




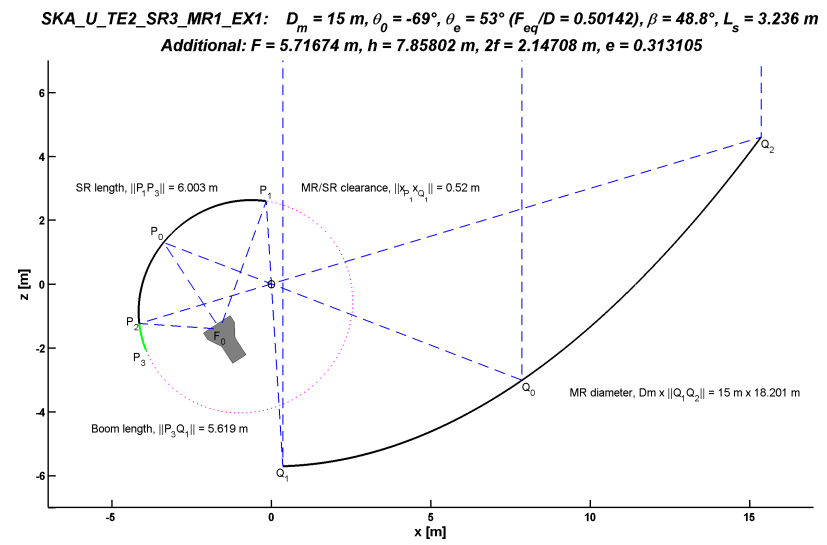

Fig. 5. Geometry of the Off-set Gregory reflector antenna of SKA1 midarray, as designed by EMSS (South Africa) [18].

where $\beta_{1}$ and $\beta_{2}$ are the weighting coefficients, and $S_{\text {appmax }}$ is the possible maximum value of $S_{\mathrm{app}}$. All of them are decided empirically. In this work, that $\beta_{1}=1, \beta_{2}=1.5$ and $S_{\text {appmax }}=10$ is chosen.

In SCA, all individuals after the first generation are grouped into several societies with the following steps. 1) Sort out all individuals depending on their fitness values in the descending order (largest to smallest). 2) Calculate the distance for all individuals of $n=2, \cdots, N$ to the 1 st individual (the best) by

$$
D(n)=\sqrt{\sum_{m=1}^{M}\left(\alpha_{m, n}-\alpha_{m, 1}\right)^{2}},
$$

where $N$ is the size of the population (the total number of individuals), $M$ the size of genes (the number of geometrical parameters), $\alpha_{m, n}$ the $m$ th geometrical parameter of the $n$th individual. 3) Set up a society boundary $\Delta D_{1}$, and group all individuals with their distance from the first individual smaller than $\Delta D_{1}$ into Society 1.4) Repeat steps 1 - 3 for the rest individuals to get the rest societies. Note that the values of $\Delta D_{i}$ have strong effect on the number of societies, and some individuals with low fitness values may not belong to any society and are thrown away.

The optimization is carried out by the following procedure: i) A population of 200 individuals is created randomly. ii) All individuals are grouped into 4 societies. iii) In each society, all individuals follow the social behaviors of the best individual randomly add space vector parameters toward the best ones. iv) After each generation, 4 elite individuals in every society are emigrated to other 3 societies, and mixed with elite individuals in those societies with a random combination coefficient on the genes. v) Continue the steps iii and iv for several generations (five generations in this project), the best individual of all societies is the final design.

Antenna petal geometries of two polarizations are optimized separately due to the fact that a slightly different geometry at the center will affect the radiation performance slightly.

An accurate analysis of the feed performance at the SKA baseline reflectors (with 18 unshaped and 5 shaped reflector designs) has been carried out after the optimization process

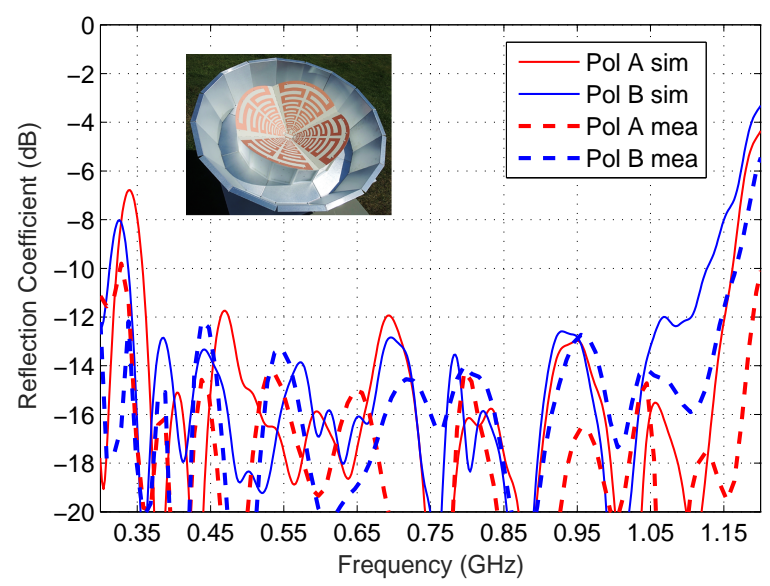

Fig. 6. Simulated and measured reflection coefficient of the present feed with the cone.

and some of these numerical results were reported in [20]. Here we present the simulations for a selected geometry with the projected main reflector of diameter $D=15 \mathrm{~m}$, the subreflector of diameter $d=6 \mathrm{~m}$, and the subtended angle of $2 \times 53^{\circ}$ (see the details in Fig. 5); the surface of the subreflector is shaped and has an extension shielding the spillover radiation in the ground region. To determine the sensitivity of the entire antenna-receiver system, we have used the $A_{e} / T_{\text {sys }}$ definition in [21] and the in-house numerical tool System Simulator [22]. This tool has been implemented in MatLab and interfaced with the reflector antenna software GRASP [23] to obtain the total antenna far-field function (based on the imported far field function data for the feed simulated in CST) and enables the post-processing of the data so as to compute the antenna directivity, SLLs, cross-polarization and noise temperature contributions due to the ground thermal noise and sky brightness. These and other noise contributions, such as those due to the ohmic losses of the balun and receiver noise are added up to calculate the resultant $T_{s y s}$ and the overall system sensitivity.

\section{Simulated and Measured Results}

The final optimized Eleven feed has a size of $745 \mathrm{~mm}$ in diameter and $241 \mathrm{~mm}$ in height with the petals on Rogers $1.5 \mathrm{~mm}$ thick RO4003 boards. The manufactured prototype of the feed with a metal cone which has a size of $1200 \mathrm{~mm}$ in diameter and $250 \mathrm{~mm}$ in height is shown in Fig. 1.

The simulated and measured reflection coefficients of the antenna are shown in Fig. 6, and are below $-12 \mathrm{~dB}$ over the band for both polarizations. Fig. 7 shows the measured mutual couplings between the two orthogonal polar ports. As expected, the isolation is very large, more than $55 \mathrm{~dB}$ over the frequency band. This confirms that the new center-bridge geometry is an improvement over the previous design.

The radiation performance was measured at Sigma Conductivity $A B$ with a Satimo Stargate SR-64 system using multiprobe measurement technology [24], see Fig. 8. Fig. 9 shows the measured co- and cross-polar radiation patterns in the $\varphi=45^{\circ}$ plane over $0.35-1.05 \mathrm{GHz}$ for both polarizations. 


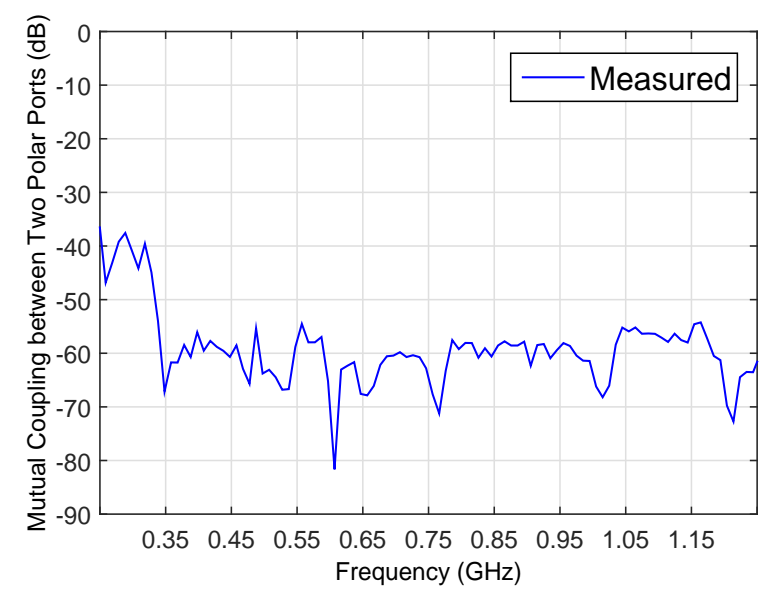

Fig. 7. Measured mutual coupling between two polarization ports

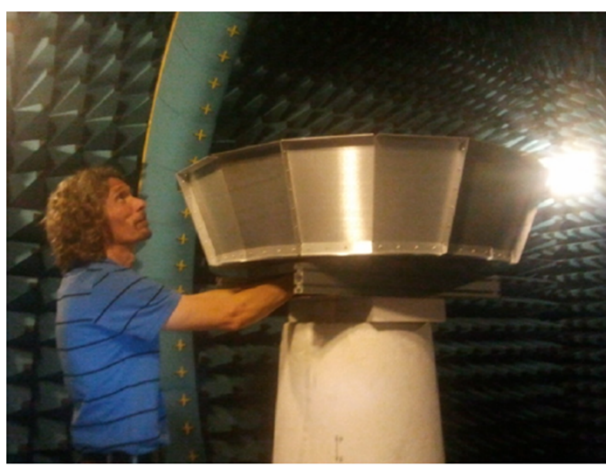

Fig. 8. Measurement set-up at Sigma Conductivity AB

It can be seen that the co-polar $-10 \mathrm{~dB}$ beamwidth changes within $110^{\circ} \pm 10^{\circ}$, nearly constantly. The simulated and measured maximum relative cross polar levels in the radiation pattern is shown in Fig. 10. The simulated relative cross-polar level is below $-15 \mathrm{~dB}$, while the measured is mainly below -15 $\mathrm{dB}$ with a few exceptions of a bit above $-15 \mathrm{~dB}$. The reason for that is mainly the mechanical mounting asymmetry of the petals of the antenna, which can be seen from the measured radiation pattern shown in Fig. 9, where the asymmetry is clearer in the cross-polar patterns. It is worth mentioning that for identically orthogonal polarized BOR $_{1}$ antennas (the present Eleven feed is very close to this ideal antenna), the relative cross polar level is the reciprocal of the intrinsic crosspolarization ration (IXR) [25] which is required by the SKA project to be better than $15 \mathrm{~dB}$ after reflector. The present Eleven feed fulfills this requirement with a large margin since the cross-polar level will be $6 \mathrm{~dB}$ lower after reflector [20].

The aperture efficiency and receiving sensitivity of the SKA Band 1 system (Fig. 5) with the present feed has been simulated based on the measured feed far field function by using the System Simulator (see Sec. III in [22]) which uses the definition of the sensitivity in [21] and is interfaced with GRASP - Physical Optics (PO) tool - to compute the antenna directivity. The results are shown in Fig. 11, where the efficiency is higher than $70 \%$ at the high frequency end and $55 \%$ at the low frequency end. Radiation efficiency, a critical

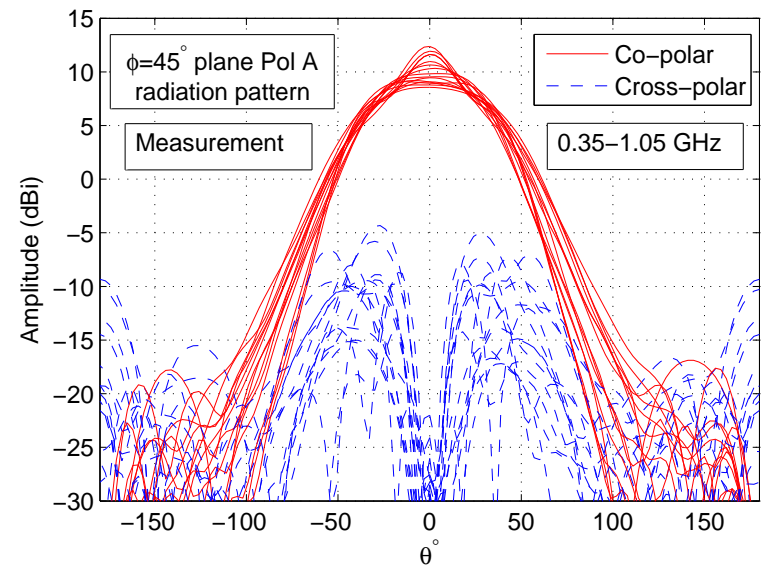

(a) Pol A

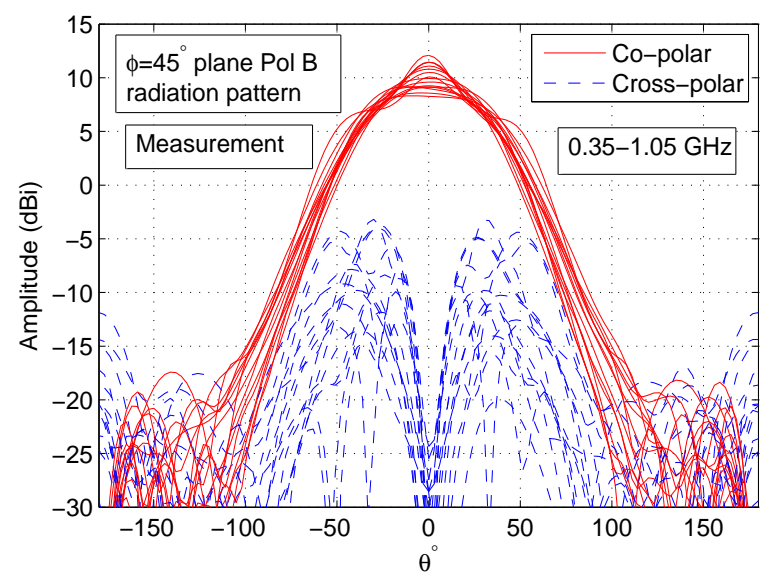

(b) Pol B

Fig. 9. Measured radiation patterns in $\varphi=45^{\circ}$ plane for (a) Pol A and (b) Pol B.

measure of the ohmic loss of the antenna in radio telescope applications, was measured also at Sigma Conductivity AB who claimed a $\pm 0.9 \mathrm{~dB}$ measurement accuracy for radiation efficiency. The simulated and measured data is shown in Fig. 12 , where the simulated is about $-0.05 \mathrm{~dB}$ (the mismatch factor is not included but the SMA connectors are included) while the measured data fluctuates between $-0.8 \mathrm{~dB}$ and $+0.4 \mathrm{~dB}$ (SMA connectors are included, which have $0.06 \mathrm{~dB}$ insertion loss from manufacture's specification), which is consistent with the claimed measurement accuracy. Therefore, we believe that the simulated data might be more accurate, and the measured data anyway indicates to some extend that the ohmic loss is small. A better way would be to use other measurement methods, such as the Reverberation Chamber measurement technology [26] with $\pm 0.5 \mathrm{~dB}$ accuracy, to obtain more accurate data.

Fig. 13 shows three possible receiver system solutions with the present feed, hybrids and low noise amplifiers (LNAs). The first one uses two pairs of single-ended LANs after the feed and two $180^{\circ}$ hybrids afterwards. For this solution the LNAs in each pair are required to be identical. The second one uses two differential LNAs directly. This solution has the lowest noise temperature if the differential LNAs have good noise 
TABLE I

RECEIVER SYSTEM NOISE TEMPERATURE

\begin{tabular}{|c|c|c|c|c|c|}
\hline Noise Component & Loss $(\mathrm{dB})$ & $\overline{T_{p h y}}$ & Noise (K) & Noise referred to input $(\mathrm{K})$ & Comment \\
\hline Feed ohmic losses & 0.05 & 300 & 3.47 & 3.47 & predicted with CST \\
\hline $\begin{array}{l}\text { Interconnect between feed } \\
\text { and hermetic feed through }\end{array}$ & 0.015 & 300 & 1.04 & 1.05 & \\
\hline $\begin{array}{l}\text { Hermatic feed } \\
\text { through }\end{array}$ & 0.047 & 300 & 3.26 & 3.31 & $\begin{array}{c}\text { Max. insertion } \\
\text { loss from data sheet }\end{array}$ \\
\hline SS cryo coax & 0.12 & 160 & 4.48 & 4.6 & $\begin{array}{c}120 \mathrm{~mm} \text { cryo cable } \\
\text { (Copper - SS, dielectric coax) }\end{array}$ \\
\hline Balun & 0.3 & 20 & 1.43 & 1.52 & $\begin{array}{l}\text { New design [12], } \\
\text { predicted with CST }\end{array}$ \\
\hline LNA & & & 3.00 & 3.41 & Expected LNA noise \\
\hline Digitizer + Backend & & & & 2.00 & \\
\hline Total Noise $(\mathrm{K})$ & & & & 19.4 & \\
\hline
\end{tabular}

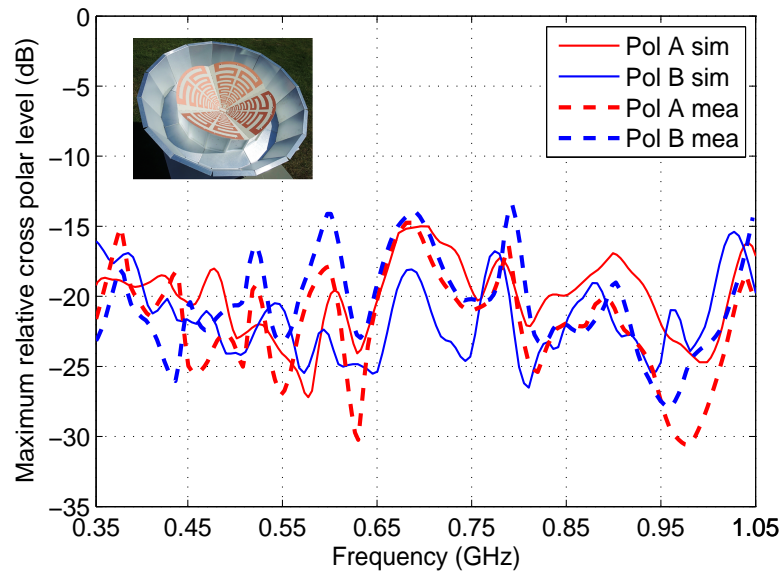

Fig. 10. Simulated and measured maximum relative cross-polar level of the present feed with cone.

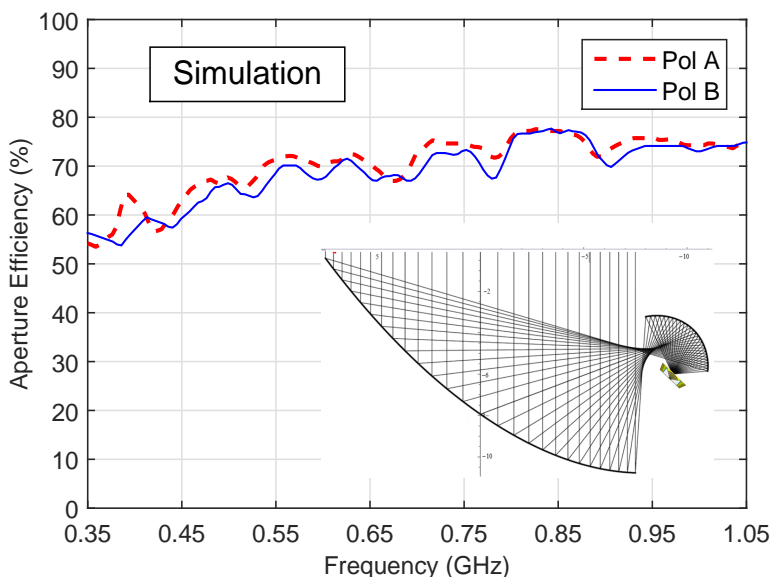

Fig. 11. Simulated aperture efficiency of the SKA1 dual reflector system with the present feed based on the measured feed radiation pattern by using the System Simulator.

performance and there is no requirement that the two dLNAs be identical (the difference between the dLNAs can be easily calibrated out). The third one consists of the Eleven feed, one dual-polarized balun [12] and two single-ended LNAs which are normally cheaper than differential LNAs. However, the

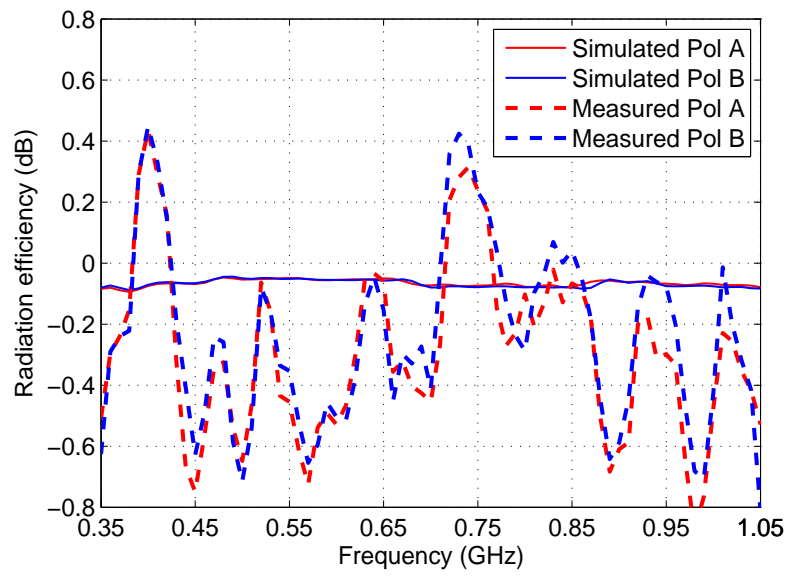

Fig. 12. Simulated and measured radiation efficiency (the mismatching factor is not included) of the dual-polarized Eleven feed

balun will increase the noise temperature by about $1.5 \mathrm{~K}$. Table I shows the noise budget for the third alternative (the worst case in terms of noise). It shows that the receiver system noise temperature is lower than $20 \mathrm{~K}$. Fig. 14 shows the calculated system sensitivity $A_{e} / T_{\text {sys }}$ when the main reflector is pointing to zenith $\theta_{p}=0$, where $A_{e}$ is the effective aperture area of the main reflector (Fig. 5) and $T_{s y s}$ is the total antenna system noise temperature, including both the antenna noise temperature $T_{a}$ and the receiver system noise temperature $T_{r}$ ( set as $20 \mathrm{~K}$ ). From the figure, it can be seen that the sensitivity is high at the higher part of the band and low in the lower part of the band. The reason for the low sensitivity in the lower band is that the beamwidth of the Eleven feed is a bit wider at low frequencies than those at high frequencies (see Fig. 9) even though they are nearly constant, which increases the noise level by picking up more noise from the ground.

\section{CONClusion}

Significant improvements to the Eleven feed technology have been achieved. With the center-bridge, the number of ports has been reduced to 4 from the previous 8 for dual polarization. By applying SCA, the reflection coefficient is improved to below $-12 \mathrm{~dB}$, the isolation between two orthogonal ports larger than $50 \mathrm{~dB}$ and the aperture efficiency above $70 \%$ 


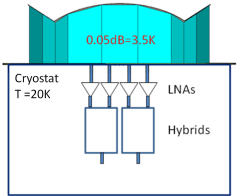

(a)

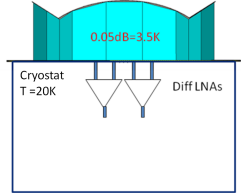

(b)

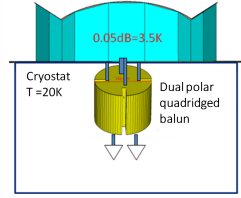

(c)
Fig. 13. Three alternatives for receiving system using the Eleven feed and LNAs: (a) Eleven feed + four single-ended LANs + two $180^{\circ}$ hybrids; (b) Eleven feed + two differential LNAs; (c) Eleven feed + balun + two singleended LNAs.

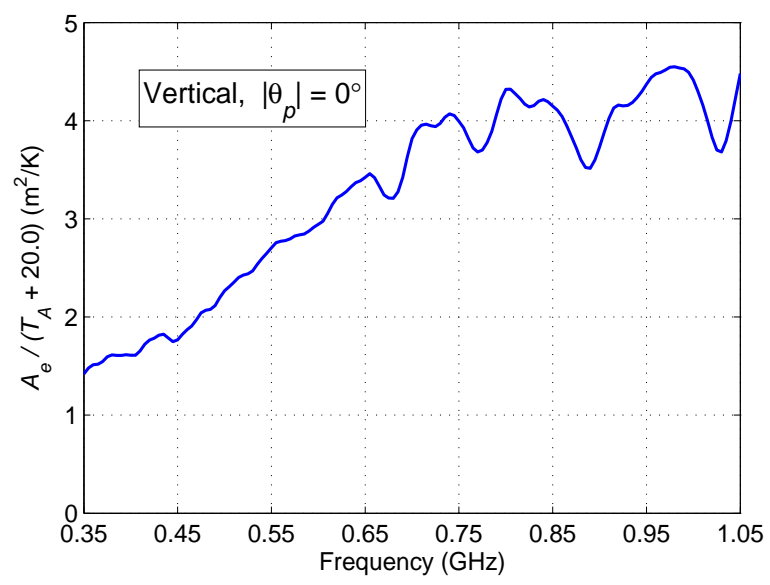

Fig. 14. Calculated sensitivity $A_{e} / T$ of the receiving system using the present Eleven feed with a balun and two LNAs (the third alternative in Fig. 13), where $A_{e}$ and $T$ are the effective aperture area and the system noise temperature respectively.

at the high part of the band. The sensitivity of the antenna system is simulated based on the measured feed's far field function.

A limiting factor for the technology presented here is that it is difficult to manufacture the tilted PCBs, especially for high frequencies.

\section{ACKNOWLEDGMENT}

This work has been supported by Swedish VR Research Infrastructures Planning Grant "Swedish contributions to the SKA radio-telescope in its pre-construction phase" (Contract No. C0546801).

\section{REFERENCES}

[1] J. Yang, M. Pantaleev, P. Kildal, B. Klein, Y. Karandikar, L. Helldner, N. Wadefalk, and C. Beaudoin, "Cryogenic 2-13 GHz Eleven feed for reflector antennas in future wideband radio telescopes," IEEE Transactions on Antennas and Propagation, vol. 59, no. 6, pp. 1918-1934, 2011.

[2] J. Yang, M. Pantaleev, P. Kildal, and L. Helldner, "Design of compact dual-polarized $1.2-10 \mathrm{GHz}$ Eleven feed for decade bandwidth radio telescopes," IEEE Transactions on Antennas and Propagation, vol. 60, no. 5, pp. 2210-2218, 2012.

[3] J. Yin, J. Yang, M. Pantaleev, and L. Helldner, "The circular Eleven antenna: a new decade-bandwidth feed for reflector antennas with a high aperture efficiency," IEEE Transactions on Antennas and Propagation, vol. 61, 2013.

[4] A. Akgiray, S. Weinreb, W. Imbriale, and C. Beaudoin, "Circular quadruple-ridged flared horn achieving near-constant beamwidth over multioctave bandwidth: Design and measurements," IEEE Trans on Antennas and Propagat., vol. 61, no. 3, pp. 1099-1108, March 2013.
[5] T. S. Beukman, M. V. Ivashina, R. Maaskant, P. Meyer, and C. Bencivenni, "A quadraxial feed for ultra-wide bandwidth quadruple-ridged flared horn antennas," in Proceedings of the 7th European Conference on Antennas and Propagation (EUCAP). IEEE, 2014.

[6] R. Gawande and R. Bradley, "Towards an ultra wideband low noise active sinuous feed for next generation radio telescopes," IEEE Trans on Antennas and Propagat., vol. 59, no. 6, pp. 1945-1953, June 2011.

[7] G. Cortes-Medellin, "Non-planar quasi-self-complementary ultrawideband feed antenna," IEEE Trans on Antennas and Propagat., vol. 59, no. 6, pp. 1935-1944, June 2011.

[8] E. Garcia and et.al, "Dyson conical quad-spiral array for vlbi2010s," in Proceedings of the 7th European Conference on Antennas and Propagation (EUCAP). IEEE, 2014.

[9] M. Ivashina, R. Bradley, M. Pantaleev, J. Yang, and C.Bencivenni, "Ultra-wideband, cryogenically-cooled reflector antenna feeds for future radio telescopes: Conical-sinuous antenna, Eleven antenna and quadridged flared horn feeds," in Proceedings of the 2014 XXXIth URSI General Assembly and Scientific Symposium (URSI GASS), Beijing, China, Aug. URSI, 2014.

[10] J. Yang, X. Chen, N. Wadefalk, and P.-S. Kildal, "Design and realization of a linearly polarized Eleven feed for 1-10 GHz," IEEE Antennas and Wireless Propagation Letters, vol. 8, pp. 64-68, 2009.

[11] H. Raza, J. Yang, and A. Hussain, "Measurement of radiation efficiency of multiport antennas with feeding network corrections," IEEE Antennas and Wireless Propagation Letters, vol. 11, pp. 89-92, 2012.

[12] J. Yang, "Preliminary design of Eleven feed for SKA band 1," in 2014 XXXIth URSI General Assembly and Scientific Symposium (URSI GASS). IEEE, 2014, pp. 1-4.

[13] X. Chen, "Study of system noise temperature from $50 \mathrm{MHz}$ to 15 GHz with application to Eleven antennas," Master Thesis, Chalmers University of Technology, 2007.

[14] A. Yasin, J. Yang, and T. Ostling, "A compact dual-band feed for reflector antennas based on choke horn and circular Eleven antenna," IEEE Transactions on Antennas and Propagation, vol. 57, no. 10, pp. 3300-3302, 2009.

[15] CST Microwave Studio; http://www.cst.com.

[16] T. Ray and K. M. Liew, "Society and civilization: An optimization algorithm based on the simulation of social behavior," IEEE Transactions on Evolutionary Computation, vol. 7, no. 4, pp. 386-396, 2003.

[17] J. Yang and P.-S. Kildal, "Optimization of reflection coefficient of large log-periodic array by computing only a small part of it," IEEE Transactions on Antennas and Propagation, vol. 59, no. 6, pp. 17901797, 2011.

[18] I. P. Theron, R. Lehmensiek, and D. de Villiers, "Towards an optics design for SKA," in IEEE AFRICON, , Mauritius, Sep. IEEE, 2013.

[19] E. G. Geterud, J. Yang, T. Ostling, and P. Bergmark, "Design and optimization of a compact wideband hat-fed reflector antenna for satellite communications," IEEE Transactions on Antennas and Propagation, vol. 61, no. 1, pp. 125-133, 2013.

[20] M. Ivashina, C. Bencivenni, O. Iupikov, and J. Yang, "Optimization of the 0.35-1.05 ghz quad-ridged flared horn and eleven feeds for the square kilometer array baseline design," in Electromagnetics in Advanced Applications (ICEAA), 2014 International Conference on. IEEE, 2014, pp. 513-516.

[21] M. V. Ivashina, R. Maaskant, , and B. Woestenburg, "Equivalent system representation to model the beam sensitivity of receiving antenna arrays," IEEE Antennas and Wirelss Propagation Letters, vol. 7, 2008.

[22] M. Ivashina, O. Iupikov, R. Maaskant, W. van Cappellen, and T. Oosterloo, "An optimal beamforming strategy for wide-field surveys with phased-array-fed reflector antennas," IEEE Trans on Antennas and Propagat., vol. 59, no. 6, pp. 1864-1875, June 2011.

[23] GRASP; http://www.ticra.com/products/software/grasp.

[24] Sigma Conductivity AB; http://sigmaconnectivity.se/.

[25] T. Carozzi and G. Woan, "A fundamental figure of merit for radio polarimeters," IEEE Transactions on Antennas and Propagation,, vol. 59, no. 6, pp. 2058-2065, 2011.

[26] Bluetest AB; http://www.bluetest.se. 